\title{
Conditional coverage and its role in determining and assessing long-term capital requirements
}

\author{
Alex Ferrer \\ Department of Quantitative Economics \\ Universidad Complutense de Madrid \\ Spain \\ J osé Casals \\ Department of Quantitative Economics \\ Universidad Complutense de Madrid \\ Spain \\ Sonia Sotoca \\ Department of Quantitative Economics \\ Universidad Complutense de Madrid \\ Spain
}

\begin{abstract}
We define the vector of conditional coverage values generated over the business cycle by a constant capital figure. Using a convenient analytical framework, we explore its properties and propose two applications based on it. For the former, we state a result that links the concepts of conditional and unconditional solvency and offers an alternative interpretation of the unconditional capital. For the latter, we propose using the minimum of the conditional coverage vector in the determination of long-term capital requirements, as well as using its minimum and its standard deviation in the long-term assessment of a given capital figure. Both applications are illustrated empirically. The entire analysis can be understood as an attempt to recognize and incorporate capital cyclicality into the measurement and analysis of default risk.
\end{abstract}

J EL Classification: C58, G21, G32

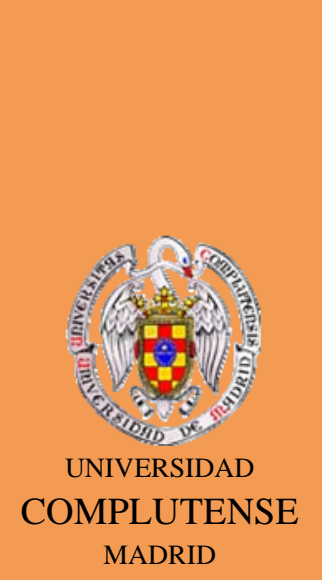

Working Paper no 1412

June, 2014

ISSN: 2341-2356

WEB DE LA COLECCIÓN: http://www.ucm.es/fundamentos-analisis-economico2/documentos-de-trabajo-del-icae Copyright (C) 2013, 2014 by ICAE.

Working papers are in draft form and are distributed for discussion. It may not be reproduced without permission of the author/s. 


\title{
Conditional coverage and its role in determining and assessing long-term capital requirements
}

- This version: June 2014 -

ICAE Working Papers

Alex Ferrer ${ }^{\dagger}$, José $_{\text {Casals }}^{\dagger}$, Sonia Sotoca ${ }^{\dagger}$

\begin{abstract}
We define the vector of conditional coverage values generated over the business cycle by a constant capital figure. Using a convenient analytical framework, we explore its properties and propose two applications based on it. For the former, we state a result that links the concepts of conditional and unconditional solvency and offers an alternative interpretation of the unconditional capital. For the latter, we propose using the minimum of the conditional coverage vector in the determination of long-term capital requirements, as well as using its minimum and its standard deviation in the long-term assessment of a given capital figure. Both applications are illustrated empirically. The entire analysis can be understood as an attempt to recognize and incorporate capital cyclicality into the measurement and analysis of default risk.
\end{abstract}

Keywords: default risk, long-term capital, unconditional capital, conditional coverage, unconditional coverage, capital cyclicality

JEL Classification: C58, G21, G32

\section{Introduction}

A whole body of literature has emerged in recent years regarding the potential cyclicality of banks' capital requirements, Drumond (2009). As discussed by Kashyap and Stein (2004), capital cyclicality may produce adverse effects on the economy and, consequently, it should be properly measured and if possible, tempered. Basel III, BCBS (2011) and BCBS (2013), has acknowledged this fact by introducing regulatory changes from Basel II, BCBS (2006).

Regarding default risk, the mechanism of capital cyclicality can be synthesized, informally, into two causal relationships: $(i)$ the worse the economic environment, the higher the default risk borne by the credit portfolio, and (ii) the higher the default risk, the higher the capital requirement.

The first relationship has been widely corroborated, Festic et al. (2011). As noted by Truck and Rachev (2005), a change in the portfolio's default risk is given by either a

\footnotetext{
†'Department of Quantitative Economics, Universidad Complutense de Madrid, Campus de Somosaguas, 28223 Madrid, Spain. Tel.: (+34) 9139423 83. Email addresses: af.econres@gmail.com (A.Ferrer),jmcasals@ccee.ucm.es (J.Casals), sotoca@ccee.ucm.es (S. Sotoca).

$\S$ Corresponding author.
}

change in its exposure profile (e.g., debtors moving from high-quality ratings to low-quality ones) or a change in the probability of default (PD) of a given type of debtor. In this paper, we focus exclusively on the latter. For this purpose, we assume a given and fixed credit investment exposition that is subject to a wide range of economic scenarios. Such scenarios give rise to different PD distributions and therefore different degrees of default risk.

The second relationship is the natural consequence of having to satisfy a certain solvency condition in every point of the business cycle. Thus, if capital is intended to cover losses up to a certain coverage level that remains the same over time, then if default risk increases, capital must increase to guarantee that coverage. Correspondingly, if the bank holds a constant amount of capital over time, then what changes is its conditional coverage, which is the coverage offered by the constant capital at a given stage of the business cycle. This dual view of cyclicality is the one we focus on throughout the paper.

Therefore, we study capital cyclicality in terms of the conditional coverage achieved over time by a constant capital under a framework where only probabilities of default vary from the hand of the economy.

This approach allows us to explore capital cyclicality from a perspective different from that focused on cyclical capital requirements caused by cyclical migrations, which 
is the case of interest in the regulatory framework ${ }^{1}$ and therefore has received most of the attention and shaped most of the discussion, as observed by Drumond (2009).

Moreover, when the focus is placed on the capital cyclicality caused by the PD, Koopman et al. (2005) and Rösch and Scheule (2010), or when coverage cyclicality is studied, Peura and Jokivuolle (2004), the main concern is the analysis of the previous causal relationships - i.e., the mechanism underlying capital cyclicality. On the contrary, we are not interested in capital cyclicality itself but in its potential uses. Thus, the objective of this paper is not to obtain evidence of capital cyclicality, Ayuso et al. (2004) and Jokipii and Milne (2008); discuss its theoretical roots, Heid (2007) and Zhu (2008); or study mitigation strategies, Gordy and Howells (2006) and Repullo et al. (2010). Instead, we seek to enhance default risk management by taking advantage of its existence, especially as regards longterm capital requirements. That is, we are interested in determining and assessing long-term capital requirements through an approach that takes into account the cyclical nature of default risk.

Our analysis adds to the existing literature in two ways.

Theoretically, we introduce and discuss the vector of conditional coverage values generated by a constant capital during the business cycle, on which the rest of the paper relies. Under a specific framework, we state a new result that links this vector with the corresponding unconditional coverage, showing that the average of the former equals the latter. This relationship ties the concepts of conditional and unconditional solvency and offers an alternative interpretation of the unconditional capital.

Methodologically, we introduce and discuss two applications of the conditional coverage vector in the long-term capital determination and assessment processes. For the former, we propose a solvency condition based on the minimum conditional coverage. That is, the long-term capital must be such that the all the components of the conditional coverage vector are equal to, or greater than, a pre-defined target. This is a novel approach to determining long-term capital requirements as the solvency condition does not rely on the unconditional loss distribution. For the capital assessment process, we propose evaluating the long-term solvency offered by a given capital not only in terms of its unconditional coverage, which is the standard approach, but also in terms of the minimum and the standard deviation of the conditional coverage vector. This means adding metrics of resilience and stability to the standard measure of long-term solvency.

We complete the analysis with an empirical exercise based on American data for six different types of credit products. Results show that conditional coverage follows cyclical and asymmetric behavior, that a significant down-

\footnotetext{
${ }^{1}$ Because the regulatory framework assumes a through-the-cycle PD, which takes a long-term average instead of its point-in-time value, cyclical movements in the regulatory capital are mainly driven by cyclical rating migrations.
}

fall occurred during the Great Recession, and that both the minimum and the standard deviation of the conditional coverage vector are useful metrics for screening portfolios in terms of long-term solvency.

The rest of the paper is set up as follows. Section 2 presents the analytical framework on which the rest of the paper relies. Section 3 introduces the concept of conditional coverage and the theoretical result based on it. Section 4 and Section 5 present and discuss our alternative methods of determining and assessing long-term capital requirements, respectively. Section 6 contains the empirical analysis and Section 7 provides a conclusion. Appendix A presents the proof for the theoretical result stated in Section 3.

\section{Framework}

\subsection{Loss model}

Time is measured discretely at regular intervals that, for simplicity, match the time horizon used by the bank to measure the default risk of its credit portfolio ${ }^{2}$. In practice, this time horizon is usually a year, but no specific assumptions about it are done. We assume that the only source of loss for the bank is its credit portfolio. With the purpose of dealing with a stylized mathematical framework, but without undermining the generality of the discussion, we consider a reduced, although standard, structure for the credit portfolio and its loss distribution, see Gordy (2000) and Frey and McNeil (2003).

The portfolio is formed by $N$ risk units, which are homogeneous groups of debtors whose exposure is assumed constant and infinitely fine-grained, Gordy (2003), so there is no need to characterize debtors individually ${ }^{3}$.

Thus, there is a vector $\boldsymbol{e}=\left(e^{1}, \ldots, e^{N}\right), e^{j}>0, j=$ $1, \ldots, N$, representing the net exposure of each risk unit. In order to keep the notation clean, we do not add any temporal subscript indicating the current period.

The loss experienced by the bank during the time horizon is then a fraction of $E=\sum_{j=1}^{N} e^{j}$. For simplicity, the traditional distinction between expected loss - to be absorbed by provisions - and unexpected loss - to be absorbed by equity - vanishes, and the focus is placed on the total volume of loss the bank is able to absorb with its own resources, which we denote as capital.

Hence, the bank's objective is to hold capital $\eta, 0 \leq$ $\eta \leq \sum_{j=1}^{N} e^{j}$, large enough to guarantee a pre-defined coverage, or solvency, condition depending on $\boldsymbol{e}$ and the loss distribution that it generates.

\footnotetext{
${ }^{2}$ However, our analysis can easily be extended to the multi-period framework, Duffie et al. (2007).

${ }^{3}$ Although this assumption is analytically convenient, it does not affect the conclusions at all, since idiosyncratic risk is not tied to the business cycle.
} 
Given $\boldsymbol{e}$, we assume that the portfolio loss distribution, $L$, is given by:

$$
L=\sum_{j=1}^{N} L^{j}=\sum_{j=1}^{N} e^{j} F^{j}
$$

where $F^{j}$ is the PD of risk unit $j$ during the time horizon. $F^{j}$ is a continuous random variable with support in $(01)$, with $\boldsymbol{F}=\left(F^{1}, \ldots, F^{N}\right)$ being the continuous multivariate PD random vector with support in $\left(\begin{array}{ll}0 & 1\end{array}\right)^{N}$.

Different $\boldsymbol{F}$ distributions result in different $L$ distributions. Throughout the paper, we consider two alternatives.

On the one hand, the conditional PD distribution, $\boldsymbol{F}_{t}=$ $\left(F_{t}^{1}, \ldots, F_{t}^{N}\right) . \quad \boldsymbol{F}_{t}$ reflects the economic environment prevailing in $t$ and is identified on the basis of the vector $\boldsymbol{h}_{t}=\left(h_{t}^{1}, \ldots, h_{t}^{N}\right)$ of observed hazard rates of the portfolio, where $h_{t}^{j}$ is the realized default rate for risk unit $j$ in period $t$. It follows that $\boldsymbol{F}_{t} \sim \boldsymbol{H}_{t}$, with $\boldsymbol{H}_{t}=\left(H_{t}^{1}, \ldots, H_{t}^{N}\right)$ being the conditional distribution of $\boldsymbol{h}_{t}$ in $t$ given all available information up to $t-1$. This means that identifying $\boldsymbol{F}_{t}$ requires previously identifying a dynamic model for $\boldsymbol{h}_{t}$, see Pesaran et al. (2006).

On the other hand, the unconditional PD distribution, $\boldsymbol{F}^{*}=\left(F^{* 1}, \ldots, F^{* N}\right) . \boldsymbol{F}^{*}$ is a $\mathrm{PD}$ distribution not subject to any specific economic scenario. As an unconditional PD model, we consider that proposed by Ferrer et al. (2014). That is, $\boldsymbol{F}^{*}$ is defined as the equally weighted mixture, or simply, mixture, of the conditional PD distributions $\boldsymbol{F}_{t}=\left(F_{t}^{1}, \ldots, F_{t}^{N}\right)$ included in a time window large enough to properly capture the long-term behavior of the portfolio's default risk. This time window should include both recessions and expansions to avoid biases. We frequently refer to it throughout the paper as the full-business-cycle time window to emphasize this alleged feature. We denote it by the stint $t=1, \ldots, T$, so that it has length $T$. Therefore, $\boldsymbol{F}^{*}$ is the mixture of the conditional distributions $\boldsymbol{F}_{t}, t=1, \ldots, T$. This approach is significantly different from the traditional static formulations, like the model of Vasicek, Vasicek (2002), which underpins the regulatory framework.

\subsection{Determining long-term capital requirements}

Determining $\eta$ requires defining a solvency condition to be met through a capital determination process. For the former, we consider as baseline a common framework in the literature for long-term capital requirements: $\eta$ is the Value at Risk (VaR) of the unconditional loss distribution at the coverage level $u, 0<u<1$. That is, $\eta$ is such that:

$$
u=P(L \leq \eta)
$$

for a given $u$ and with $L$ being the loss distribution of Eq. 1 taking $\boldsymbol{F}^{*}$ as the PD distribution. From now on, $L$ represents an unconditional loss distribution and $u$ an unconditional coverage level ${ }^{4}$.

\footnotetext{
${ }^{4} \eta$, however, only represents a long-term capital figure, which will be unconditional if it has been derived from an unconditional loss
}

According to this basic framework, the process for determining $\eta$ follows the next steps:

1. Defining a target value for the unconditional coverage level, $u$.

2. Fitting a dynamic model to $\boldsymbol{h}_{t}=\left(h_{t}^{1}, \ldots, h_{t}^{N}\right)$.

3. Defining the full-business-cycle time window, $t=$ $1, \ldots, T$.

4. Identifying the collection of conditional PD distributions $\boldsymbol{F}_{t}=\left(F_{t}^{1}, \ldots, F_{t}^{N}\right), t=1, \ldots, T$, in terms of $\boldsymbol{h}_{t}=\left(h_{t}^{1}, \ldots, h_{t}^{N}\right)$.

5. Forming $\boldsymbol{F}^{*}=\left(F^{* 1}, \ldots, F^{* N}\right)$ as the mixture of the conditional PD distributions $\boldsymbol{F}_{t}, t=1, \ldots, T$.

6. Characterizing $L$ in terms of $\boldsymbol{F}^{*}$ and the vector $\boldsymbol{e}=$ $\left(e^{1}, \ldots, e^{N}\right)$ according to Eq. 1.

7. Obtaining the capital requirement as the value $\eta$ satisfying $u=P(L \leq \eta)$.

We will refer to this process as the standard long-term capital determination process. There are two points worth mentioning.

Regarding Step 1, $u$ is chosen by the shareholders according to their risk appetite: The higher the coverage level, the higher the bank's solvency, but also the lower the bank's efficiency. Therefore, a trade-off must be elucidated. Although some theoretical frameworks of optimizing behavior have been proposed, like those of Estrella (2004) and Elizalde and Repullo (2007), in practice, shareholders usually follow a simpler approach. As noted by Carey (2002), they first choose a target rating from the scale of a rating agency, and then define $u$ according to this target rating. For example, if shareholders want an AA rating, and such a rating has a long-term (average over time) PD of 0.001, then ${ }^{5} u=99.9$.

Regarding Step 6 and Step 7, the estimation of $\eta$, which is the estimation of the $u$ percentile of $L$, can be solved either by analytical approximation, Glasserman (2004), or by Monte Carlo simulation, Glasserman et al. (2008).

\subsection{Assessing long-term capital requirements}

Although the standard process described before states a causal relationship - $u$ determines $\eta$ given $L$ - these two parameters, $u$ and $\eta$, can also be seen as a pair just linked by the condition $u=P(L \leq \eta)$. That means that two interpretations are valid: The capital that achieves a given unconditional coverage level, or the unconditional coverage level achieved by a given capital figure. The former emanates from the capital determination process, while the latter is related to the capital assessment process. More generally, by capital assessment process we mean evaluating a given capital $\eta$ in terms of a particular solvency condition. $\eta$ can be the capital that the bank actually

\footnotetext{
distribution.

${ }^{5}$ Throughout the paper, coverage values are expressed in percentage points.
} 
holds after introducing discretionary capital buffers, a hypothetical capital estimate, or, in general, any capital figure whose long-term solvency is worth evaluating regardless of the logic followed to determine it.

From a long-term perspective, the standard approach to the assessment of $\eta$ is similar to that used for its determination. In fact, the process is the same as that described in Section 2.2 except for Step 1, where defining $u$ must be replaced with defining $\eta$, because now $u$ is an output and $\eta$ an input. Similarly, in Step 7 the condition $u=P(L \leq \eta)$ must now be solved for $u$ in terms of $\eta$, and not the other way around.

The resulting $u$ can be used by the bank for its internal solvency and risk appetite analysis. It can also be reported to supervisor authorities as part of the regulatory examination, and to investors and rating agencies as part of the market discipline policies.

\section{Conditional coverage}

\subsection{Conditional coverage vector}

Given $\eta, \boldsymbol{e}=\left(e^{1}, \ldots, e^{N}\right)$ and the conditional distribution $\boldsymbol{F}_{t}$, it is natural to define the concept of conditional coverage $u_{t}$ as follows:

$$
u_{t}=P\left(L_{t} \leq \eta\right)
$$

where $L_{t}$ is the conditional loss distribution, given by Eq. 1, taking $\boldsymbol{F}_{t}$ as the PD distribution.

$u_{t}$ represents the coverage that $\eta$ offers at a given point of the cycle, that is, at a given, and observed, economic scenario. Therefore, $u_{t}$ is a measure of conditional solvency in the same way $u$ is a measure of unconditional solvency. Since $L_{t}$ is effectively faced by the bank at each period $t$, while $L$ is just a synthetic loss distribution, it could be said that conditional solvency is a much more real concern.

$\boldsymbol{h}_{t}$ and $u_{t}$ are expected to exhibit a close and opposite movement driven by the economic cycle. Thus, as observed by Jiménez and Mencía (2009), the mass of $L_{t}^{j}$ is expected to present a cyclical shift, moving to the right in recession times and to the left in expansion times. Under a constant capital $\eta$, this effect leads to higher values of $u_{t}$ during expansions and lower ones during recessions, which is just the opposite behavior of $\boldsymbol{h}_{t}$.

Obtaining the conditional coverage $u_{t}$ for each period of the time window used to form $\boldsymbol{F}^{*}$ gives rise to the conditional coverage vector $\boldsymbol{u}=\left(u_{1}, \ldots, u_{T}\right)$. This vector is the core of the paper and can be seen as the collection of conditional coverage values that the bank would experience during a full-business-cycle time window if $\boldsymbol{e}$ and $\eta$ were kept constant. Therefore, in spite of containing conditional information, $\boldsymbol{u}$ offers a long-term view of bank solvency.

\subsection{Relationship with the unconditional coverage}

Under the model assumed for $\boldsymbol{F}^{*}, \boldsymbol{u}$ and $u$ are linked by a linear condition, as stated in Proposition A.

\section{Proposition A.}

Let $L$ be the unconditional loss distribution given by

$$
L=\sum_{j=1}^{N} L^{j}=\sum_{j=1}^{N} e^{j} F^{* j}
$$

with $\boldsymbol{F}^{*}=\left(F^{* 1}, \ldots, F^{* N}\right)$ formed as the mixture of the conditional PD distributions $\boldsymbol{F}_{t}=\left(F_{t}^{1}, \ldots, F_{t}^{N}\right), t=1, \ldots, T$.

Given $\eta, 0<\eta<\sum_{j=1}^{N} e^{j}$, it is satisfied that

$$
u=\frac{1}{T} \sum_{t=1}^{T} u_{t}
$$

where $u=P(L \leq \eta)$ and $u_{t}=P\left(L_{t} \leq \eta\right)$, being

$$
L_{t}=\sum_{j=1}^{N} L_{t}^{j}=\sum_{j=1}^{N} e^{j} F_{t}^{j}
$$

Proof.

See Appendix A.

Proposition A states that the unconditional coverage is the average of the conditional coverage values for any given time window, capital, and exposure profile. This is a new result whose relevance stems from the clear and simple relationship it provides between unconditional and conditional solvency. It also outlines the analytical tractability of the model considered for $\boldsymbol{F}^{*}$, as opposed to the static formulations.

There are two immediate implications of Proposition A.

First, in practice, $u_{t}$ will be lower than $u$ in some periods, especially during recession periods. This is, arguably, what keeping a constant capital is all about: Obtaining a lower coverage in recessions and a higher one in expansions, with the average of these conditional coverage values equal to the unconditional one, according to Proposition A. In other words, $\eta$ has to meet an unconditional restriction, not a conditional one. However, if $u_{t}<<u$ the market may fear that the actual value of $\eta$ is less than reported. If this occurs, a confidence crisis regarding the solvency of the bank may appear and trigger rating downgrades.

Second, Eq. 5 offers a different interpretation of the unconditional condition to be met by $\eta$. Thus, instead of understanding the unconditional capital as the capital that guarantees a target coverage level $u$ under the unconditional loss distribution, it can be seen as the capital that guarantees that the average of the coverage values obtained under a varied collection of conditional loss distributions, which represent different stages of the business 
cycle, equals $u$. Since the concept of conditional loss distribution may be easier to understand than that of unconditional loss distribution ${ }^{6}$, especially by those not interested in the more technical details, like the senior management, this approach to the concept can be convenient for communication purposes.

\section{Using the conditional coverage vector in the capital determination process}

\subsection{Proposal}

The long-term capital determination process described in Section 2 is based on the unconditional loss distribution and the unconditional coverage level related to it. We propose an alternative process relying on the conditional loss distributions included in the time window and the vector $\boldsymbol{u}=\left(u_{1}, \ldots, u_{T}\right)$ of conditional coverage levels related to them. This means defining $\eta$ in terms of some function of $\boldsymbol{u}$ and not in terms of $u$. To this end, we propose using its minimum, which can be understood as a downturn coverage level, $u^{d t}=\min \left\{u_{1}, \ldots, u_{T}\right\}$.

Under this choice, the proposed capital determination process follows the next steps:

1. Defining a target value for the downturn coverage level, $u^{d t}$.

2. Fitting a dynamic model to $\boldsymbol{h}_{t}=\left(h_{t}^{1}, \ldots, h_{t}^{N}\right)$.

3. Defining the full-business-cycle time window, $t=$ $1, \ldots, T$.

4. Identifying the collection of conditional PD distributions $\boldsymbol{F}_{t}=\left(F_{t}^{1}, \ldots, F_{t}^{N}\right), t=1, \ldots, T$, in terms of $\boldsymbol{h}_{t}=\left(h_{t}^{1}, \ldots, h_{t}^{N}\right)$.

5. Characterizing $L_{t}, t=1, \ldots, T$, in terms of $\boldsymbol{F}_{t}$ and the vector of total exposures $\boldsymbol{e}=\left(e^{1}, \ldots, e^{N}\right)$.

6 . Obtaining the capital requirement as the value $\eta$ satisfying $u^{d t}=\min _{t}\left\{P\left(L_{t} \leq \eta\right)\right\}$.

\subsection{Discussion}

The most striking difference between our proposal and the standard determination processes is that the step devoted to characterizing $L$ disappears under the former, as there is no need to use the unconditional loss distribution at any stage of the process. In other words, the proposed capital determination process attempts to provide a longterm capital figure without relying on the unconditional measurement.

This is a remarkable feature. By defining the solvency condition in terms of $\boldsymbol{u}$, which contains conditional information but reflects a full-business-cycle time window, we have disjoined two concepts usually presented as one:

\footnotetext{
${ }^{6}$ As noted by Ferrer et al. (2014), the concept of unconditional PD distribution, and therefore unconditional loss distribution, is tricky, especially if $\boldsymbol{h}_{t}$ is not stationary. The conditional PD distributions, by contrast, can always be properly defined regardless of the stationarity of $\boldsymbol{h}_{t}$
}

Long-term capital and unconditional capital. The former is now more general than the latter and can be understood, informally, as a capital estimate derived from a solvency condition that is not related to any economic scenario, and therefore to any conditional loss distribution. On the contrary, it is derived from a general economic environment observed during a full-business-cycle time window. Such time window is, in fact, what the standard process, based on the unconditional loss distribution, and the proposed process, based on the $T$ conditional loss distributions, have in common, although they make use of it in a different way.

Three additional points can be made about the proposed determination process.

First, it reduces the information contained in $\boldsymbol{u}$ to a one-dimension variable, $u^{d t}$. This is due to the fact that there is no univocal relationship between $\eta$ and $\boldsymbol{u}$. In other words, for any arbitrary $\eta$, there is always a vector $\boldsymbol{u}$, but the inverse is not true. Thus, shareholders cannot, for example, define a target value for both the minimum and the maximum conditional coverage to be satisfied by the same constant capital $\eta$. The alternative to this limitation is, of course, to use a dynamic capital schedule oriented to achieve a target conditional coverage at every point of the cycle. Such target can depend on the prevailing economic conditions, with a lower level in recessions than in expansions, as suggested by Repullo (2013), or, on the contrary, be constant over time, which would exacerbate the cyclicality problem.

Second, it produces a capital figure that is influenced, as also happens with the standard determination process, by the choice of time window. Thus, for example, if a significant crisis is included or removed from the time window, the minimum of $\boldsymbol{u}$ changes and so does $\eta$. Conversely, adding or removing a period related to a moderate recession will not change it at all if the recession periods already included in the time window are more severe.

Finally, it also helps shareholders to better understand, and hence better manage, the risk of suffering a confidence crisis due to a downfall in conditional coverage. Thus, the proposed determination process is specifically oriented to achieve a coverage level equal to, or greater than, a target value at every period of the full-business-cycle time window, including recession periods.

This point could suggest that we are not obtaining a long-term capital estimate but a stressed one, as in the analysis of Varotto (2012). However, we do not characterize $u^{d t}$ in terms of any specific economic scenario, either observed, like the Great Recession, or hypothetically defined, like those considered in stress test exercises. On the contrary, $u^{d t}$ is defined as the minimum of $\boldsymbol{u}$, which means that its economic interpretation depends on the choice of full-business-cycle time window and the behavior of the hazard rates series during it.

Implementing the proposed determination process requires $(i)$ defining a target value for $u^{d t}$, Step 1, and (ii) solving $\eta$ for the condition $u^{d t}=\min _{t}\left\{P\left(L_{t} \leq \eta\right)\right\}$, Step 
6.

Regarding Step 1, the rating method introduced in Section 2 for the standard process can be adapted to be used in the proposed process. Thus, instead of considering the average of the rating hazard rate series during the time window, shareholders should now resort to its maximum, $h^{R}$. Thus, the target value would be:

$$
u^{d t}=1-h^{R}
$$

With respect to the calculation of $\eta$, Step 6 , if $\eta_{t}$ is the conditional capital at level $u^{d t}$,

$$
u^{d t}=P\left(L_{t} \leq \eta_{t}\right)
$$

then it is clear that, under the proposed process, $\eta$ is given by

$$
\eta=\max \left\{\eta_{1}, \ldots, \eta_{T}\right\}
$$

Under this definition $\eta \geq \eta_{t}, t=1, \ldots, T$, and therefore $u_{t} \geq u^{d t}, t=1, \ldots, T$, with these inequalities becoming equalities in one period at least.

This result suggests an increase in the complexity of the determination process, since the percentile estimation, solved either by Monte Carlo simulation or analytical approximation, must be repeated for each conditional loss distribution instead of being conducted only for the unconditional loss distribution. There are, however, some extenuating conditions.

On the one hand, in practice, only a small number of conditional loss distributions will be sufficiently shifted to the right to generate the minimum conditional coverage of the time window. So if the hazard rate series present a clear and common cyclical pattern, the conditional loss distributions related to the growth part of the business cycle can be discarded in advance. This kind of filter can save a large part of the total computational cost.

On the other hand, the target values for $u^{d t}$ are expected to be lower than those for $u$ because the criterion to be met by $\eta$ is more stringent under the former - a minimum coverage must now be achieved at each period of the time window. This fact allows for a lower number of Monte Carlo simulations or better accuracy of the analytical approximation because $\eta$ is a less extreme percentile.

\section{Using the conditional coverage vector in the capital assessment process}

\subsection{Proposal}

Similar to the capital determination process, different long-term interpretations of the solvency that is offered by a given $\eta$ can be obtained in the capital assessment process. The word "interpretation" outlines the fact that $\eta$ is evaluated under different solvency conditions related to different views about the concept of long-term solvency.

Before presenting our proposed assessment, it is worth noting that the fact that $\eta$ can be interpreted in various ways could suggest that the capital determination process is unimportant: Both the unconditional and downturn processes can lead to any given $\eta, 0<\eta<E$, with a clever choice of $u$ or $u^{d t}$. Therefore, the only relevant feature of $\eta$ would be that it is constant.

On the contrary, the logic that supports $\eta$ matters: As happens in any other field, decision-making in risk management is influenced by the framework that is assumed. Thus, the solvency condition to be met by $\eta$ shapes the shareholders' final decision about how much capital to hold. Moreover, in purity, first the bank sets the target coverage level according to some rationale and then obtains the capital that satisfies it, rather than the other way around. In other words, the capital determination process is intended to provide a capital estimate, not to justify the existing capital by defining a convenient target value for either $u$ or $u^{d t}$.

Thus, any given capital $\eta$ generates an unconditional coverage, $u$, derived from the unconditional loss distribution, $L$, and also a minimum conditional coverage, $u^{d t}$, that is derived from the collection of conditional loss distributions $L_{t}, t=1, \ldots, T$. The long-term assessment of $\eta$ could then be given by the pair formed by $u$ and $u^{d t}$. However, the vector $\boldsymbol{u}$ comprises more relevant information than just its average and its minimum. More specifically, since $u_{t}$ is expected to vary cyclically over the time window, a measure of the variability of $\boldsymbol{u}$ can be useful for evaluating the stability of the conditional solvency offered by $\eta$. We consider the standard deviation of $\boldsymbol{u}, s^{\boldsymbol{u}}$, for this purpose.

Therefore, we propose assessing the long-term solvency of a given capital $\eta$ by means of three indicators: $u, u^{d t}$, and $s^{u}$. This means adding metrics of resilience, $u^{d t}$, and stability, $s \boldsymbol{u}$, to the standard measure of long-term solvency, $u$.

\subsection{Discussion}

Three points can be drawn about the use of $s^{\boldsymbol{u}}$ for measuring conditional solvency stability.

First, although derived from conditional information, it is a long-term indicator, since it reflects a full-businesscycle time window. In general, a lower value of $s^{\boldsymbol{u}}$ is preferable to a higher one because it reduces the probability of suffering a conditional coverage downfall.

Second, it is appropriate for comparing different portfolios or time windows if their corresponding capital figures all achieve the same unconditional coverage, $u$, since the mean of $\boldsymbol{u}$ is then the same. On the other hand, using $s^{\boldsymbol{u}}$ in the capital determination process would be impractical because there is nothing to guarantee that, for a given value of $s^{u}$, there is a $\eta$, or that, if it does exist, that it is unique.

Third, $s^{\boldsymbol{u}}$ is related to $u^{d t}$, since lower values of the latter are expected to cause higher values of the former. However, it captures a different feature of $\eta$ - stability instead of resilience - and, therefore, both are informative in their own. 
The triplet formed by $u, u^{d t}$, and $s^{\boldsymbol{u}}$ enhances the standard long-term capital assessment, which is focused exclusively on $u$. The bank could use this triplet for its own solvency analysis - e.g., setting its risk appetite statementor report it to the senior management, regulators, supervisors, investors, rating agencies or any other stakeholder in the same way as with $u$.

It is worth mentioning that, although stress test exercises are also oriented to the assessment of solvency from a conditional perspective, Sorge and Virolainen (2006) and Foglia (2009), our approach differ from them significantly. Thus, stress testing usually considers hypothetical, rather than observed, scenarios. Stress testing also focuses on short-term solvency and not its long-term equivalent. Further, it measures capital sensitivity under a fixed coverage target and not coverage sensitivity under a fixed amount of capital.

\section{Empirical analysis}

\subsection{Data, dynamic models and credit portfolios}

We use the same set of hazard rate series, dynamic models and portfolios than those employed by Ferrer et al. (2014).

In the case of the hazard rate series, that means using as a proxy of hazard rate series the quarterly series of charge-off provided by the FDIC ${ }^{7}$ for "Mortgages" (1-4 Family Residential Real Estate Loans), "Business" (Commercial \& Industrial Loans to U.S. Addressees), "Credit Cards" (Credit Cards), "Individuals" (Other Loans to Individuals), "Rest" (All Other Loans) and "Lease" (Lease Financing Receivables) between 1991Q1-2010Q4. We consider the stint 1991Q1-2010Q4 as the full-business-cycle time window.

Table 1 summarizes their main statistics and Figure 1 shows the six series together. They are non-stationary and exhibit a cyclical pattern with some degree of heterogeneity.

The dynamic model for each hazard rate series is ARIMA with a probit link function. That is, an univariate ARIMA model with normal innovations is fitted to each series $x_{t}^{j}=$ $N^{-1}\left(h_{t}^{j}\right)$. This formulation generates conditional distributions of the form $F_{t}^{j}=H_{t}^{j}=N\left(X_{t}^{j}\right)$, with $X_{t}^{j}$ being the conditional distribution of $x_{t}^{j}$ given all the available information up to $t-1$. Table 2 summarizes the univariate ARIMA models.

Each hazard rate series represents a stand-alone portfolio that is formed by a single risk unit, so that there are six portfolios. For simplicity, we assume that the total exposure of each portfolio satisfies $e=1$. Therefore, $L=e F=F$ has support in $(01)$. For the unconditional

\footnotetext{
${ }^{7}$ Federal Deposit Insurance Corporation, see http://www.fdic.gov/.
}

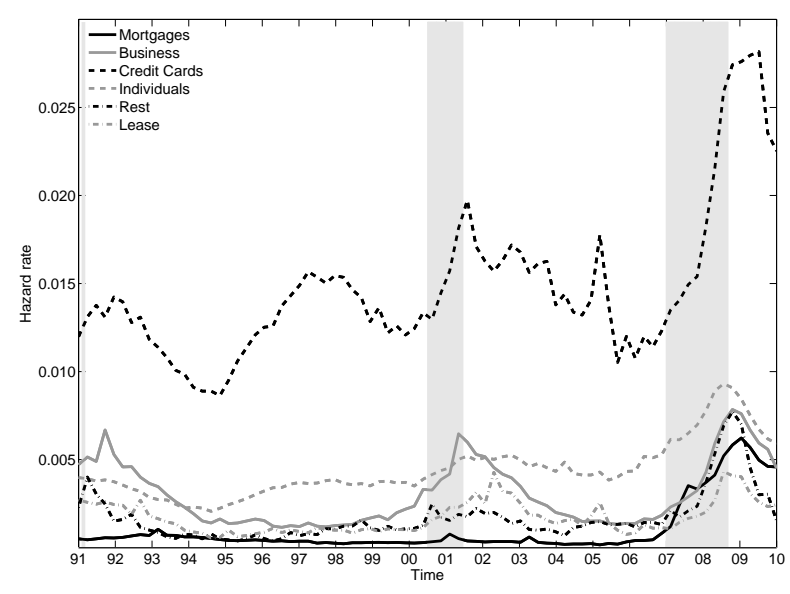

Figure 1: Hazard rates series. Shaded areas indicate U.S. recessions according to NBER (National Bureau of Economic Research).

coverage target, we use 99.9, which is the Basel coverage, as well as 99 for comparison purposes.

Based on these series, models, and portfolios, we study the behavior of $\boldsymbol{u}, u^{d t}$, and $s^{\boldsymbol{u}}$.

\subsection{Results}

Table 3 presents the unconditional capital figures for the coverage levels $u=99$ and $u=99.9$. The greater values correspond to the Credit Cards unit and, as expected, they increase significantly when passing from the former coverage level to the latter.

\begin{tabular}{lcc}
\hline \multicolumn{1}{c}{ Portfolio } & $u=99$ & $u=99.9$ \\
\hline Mortgages & 0.0070 & 0.0088 \\
Business & 0.0089 & 0.0103 \\
Credit Cards & 0.0299 & 0.0329 \\
Individuals & 0.0099 & 0.0108 \\
Rest & 0.0085 & 0.0115 \\
Lease & 0.0050 & 0.0064 \\
\hline
\end{tabular}

Table 3: Unconditional capital for the $99, u=99$, and the $99.9, u=99.9$, coverage levels.

For each portfolio, Figure 2 shows the conditional coverage series generated by the unconditional capital $\eta$ at both coverage levels. Similarly, Figure 3 compares, standardized, the hazard rate series and the conditional coverage series generated by the unconditional 99.9 capital.

These figures reveal three results.

First, $u_{t}$ follows, as expected, a cyclical pattern with higher conditional coverage values in periods of economic growth and lower ones in recessions. This result supports the use of $\boldsymbol{u}$ for capturing cyclicality. It is also consistent with the evidence presented by Koopman et al. (2005) and Rösch and Scheule (2010) for a cyclical capital under a constant coverage level.

Second, such cyclical evolution is asymmetric, with $u_{t}$ being very close to 1 in almost every period of the time 


\begin{tabular}{lccccccc}
\hline \multicolumn{1}{c}{ Series } & Mean & Std. Dev. & Min & Median & Max & JB test & ADF test \\
\hline Mortgages & 0.0010 & 0.0015 & 0.0002 & 0.0004 & 0.0062 & 0.0010 & 0.9667 \\
Business & 0.0030 & 0.0018 & 0.0012 & 0.0023 & 0.0078 & 0.0133 & 0.3653 \\
Credit cards & 0.0148 & 0.0044 & 0.0086 & 0.0138 & 0.0282 & 0.0010 & 0.8222 \\
Individuals & 0.0044 & 0.0016 & 0.0021 & 0.0039 & 0.0093 & 0.0016 & 0.7740 \\
Rest & 0.0017 & 0.0014 & 0.0004 & 0.0013 & 0.0077 & 0.0010 & 0.1569 \\
Lease & 0.0017 & 0.0009 & 0.0005 & 0.0014 & 0.0043 & 0.0075 & 0.2294 \\
\hline
\end{tabular}

Table 1: Main statistics for the hazard rate series. The p-value is presented for the Jarque-Bera test (JB test) and the Augmented Dickey-Fuller test (ADF test).

\begin{tabular}{|c|c|c|c|c|c|c|c|c|c|c|c|c|}
\hline \multirow{2}{*}{ Risk Unit } & \multicolumn{2}{|c|}{$\rho_{1}$} & \multicolumn{2}{|c|}{$\rho_{2}$} & \multicolumn{2}{|c|}{$\theta_{1}$} & \multicolumn{2}{|c|}{$\theta_{2}$} & \multirow{2}{*}{$\widehat{\sigma_{a}}$} & \multirow{2}{*}{$L B Q(16)$} & \multirow{2}{*}{$A I C$} & \multirow{2}{*}{$S B C$} \\
\hline & $\widehat{\rho_{1}}$ & $\widehat{\sigma}_{\widehat{\rho_{1}}}$ & $\widehat{\rho_{2}}$ & $\widehat{\sigma}_{\widehat{\rho_{2}}}$ & $\widehat{\theta_{1}}$ & $\widehat{\sigma}_{\widehat{\theta_{1}}}$ & $\widehat{\theta_{2}}$ & $\widehat{\sigma}_{\widehat{\theta_{2}}}$ & & & & \\
\hline Mortgages & - & - & 0.2120 & 0.1070 & - & - & - & - & 0.0670 & 0.0913 & -2.44 & -2.38 \\
\hline Business & 0.7602 & 0.1137 & - & - & 0.4882 & 0.1456 & - & - & 0.0435 & 0.4473 & -3.25 & -3.16 \\
\hline Credit Cards & - & - & - & - & - & - & - & - & 0.0359 & 0.1160 & -3.72 & -3.69 \\
\hline Individuals & 0.6230 & 0.1443 & - & - & 0.5208 & 0.2090 & -0.2886 & 0.1183 & 0.0208 & 0.1218 & -4.69 & -4.57 \\
\hline Rest & - & - & - & - & - & - & - & - & 0.0869 & 0.9121 & -1.98 & -1.95 \\
\hline Lease & - & - & - & - & - & - & - & - & 0.0712 & 0.8821 & -2.37 & -2.34 \\
\hline
\end{tabular}

Table 2: Univariate ARIMA models fitted to $x_{t}^{d}, x_{t}^{d}=x_{t}-x_{t-1}$, being $x_{t}^{d}=\rho_{1} x_{t-1}^{d}+\rho_{2} x_{t-2}^{d}-\theta_{1} a_{t-1}-\theta_{2} a_{t-2}+a_{t}$ and $V\left[a_{t}\right]=\left(\sigma_{a}\right)^{2}$. $\hat{\beta}$, estimated parameter. $\widehat{\sigma}_{\hat{\beta}}$, estimated standard deviation of $\hat{\beta}$. LBQ(16), Ljung-Box Q test p-value with 16 lags. AIC, Akaike Information Criteria. SBC, Schwarz Information Criteria.

window except during the Great Recession, when $u_{t}<<u$. This result emphasizes the severity of the Great Recession with respect to previous crises, this time in terms of conditional coverage values. Therefore, it can be considered an "acid test" for the conditional solvency that is offered by a given capital $\eta$.

It is worth noting, however, that the precise period of the Great Recession where the minimum conditional coverage is achieved differs across portfolios. This mild heterogeneity can be considered a signal of diversification if such portfolios are interpreted as risk units of the same aggregated portfolio. Thus, this result suggests that the standalone conditional coverage vectors, $\boldsymbol{u}^{j}=\left(u_{t}^{j}, \ldots, u_{t}^{j}\right)$, where $u_{t}^{j}=P\left(L_{t}^{j} \leq \eta^{j}\right)$, with $\eta^{j}$ satisfying $u=P\left(L^{j} \leq \eta^{j}\right)$, can help to identify diversification opportunities within the aggregated portfolio.

Third, during the Great Recession the 99.9 unconditional capital presents a moderate decrease in conditional coverage, while the 99 generates a sharper decline. On the contrary, during the rest of the time window, both series are very close. This effect shows that, in expansions, high conditional coverage values can be achieved with moderate unconditional coverage. However, in recessions, holding an amount of capital with a high unconditional coverage makes a real difference. In other words, since $u$ is the average of $\boldsymbol{u}$, any variation in the former means a variation in the average of the latter. The results obtained indicate that, for high values of $u$, such variation is likely to be achieved through a reduced group of elements - those related to severe recessions - and not through a harmonized variation in every element of $\boldsymbol{u}$.

Table 4 clarifies the differences in resilience during the
Great Recession by showing the value of $u^{d t}$ for each portfolio and unconditional coverage value.

\begin{tabular}{lcc}
\hline \multicolumn{1}{c}{ Portfolio } & $\eta_{99}$ & $\eta_{99.9}$ \\
\hline Mortgages & 69.68 & 95.96 \\
Business & 65.96 & 95.09 \\
Credit Cards & 76.96 & 97.21 \\
Individuals & 51.15 & 94.26 \\
Rest & 65.69 & 95.57 \\
Lease & 77.55 & 97.17 \\
\hline
\end{tabular}

Table 4: Downturn coverage generated by the capital figures of Table $3, \eta_{99}$ and $\eta_{99.9}$, which have been obtained for the 99 and 99.9 unconditional coverage levels, respectively.

A marked decrease in coverage can be observed, especially if the results are read in terms of $\alpha=1-u$ and $\alpha^{d t}=1-u^{d t}$, which can be interpreted as the unconditional and conditional PD of the bank. Thus, for example, for the Business portfolio $\alpha^{d t}$ is approximately 34 times higher than $\alpha$ for $u=99$, and 49 times for $u=99.9$. Therefore, banks may experience sharp downfalls in conditional coverage even if they present a sound long-term solvency, which is a threat worth considering.

Table 4 also suggests that achieving a value of $u^{d t}$ similar to those considered for $u, 99$ and 99.9, would require a significant increase in $\eta$. Table 5 , which presents those increases, confirms this hypothesis.

Given a target for $u^{d t}$, Table 5 shows that there is not a clear relationship between the initial value presented by $u^{d t}$ and the required increase in $\eta$ that achieves such target. In general, the increase will depend on the shape of $L$, or, given its condition of mixture, the conditional loss distributions that form it. For instance, if all $L_{t}, t=1, \ldots, T$, are 

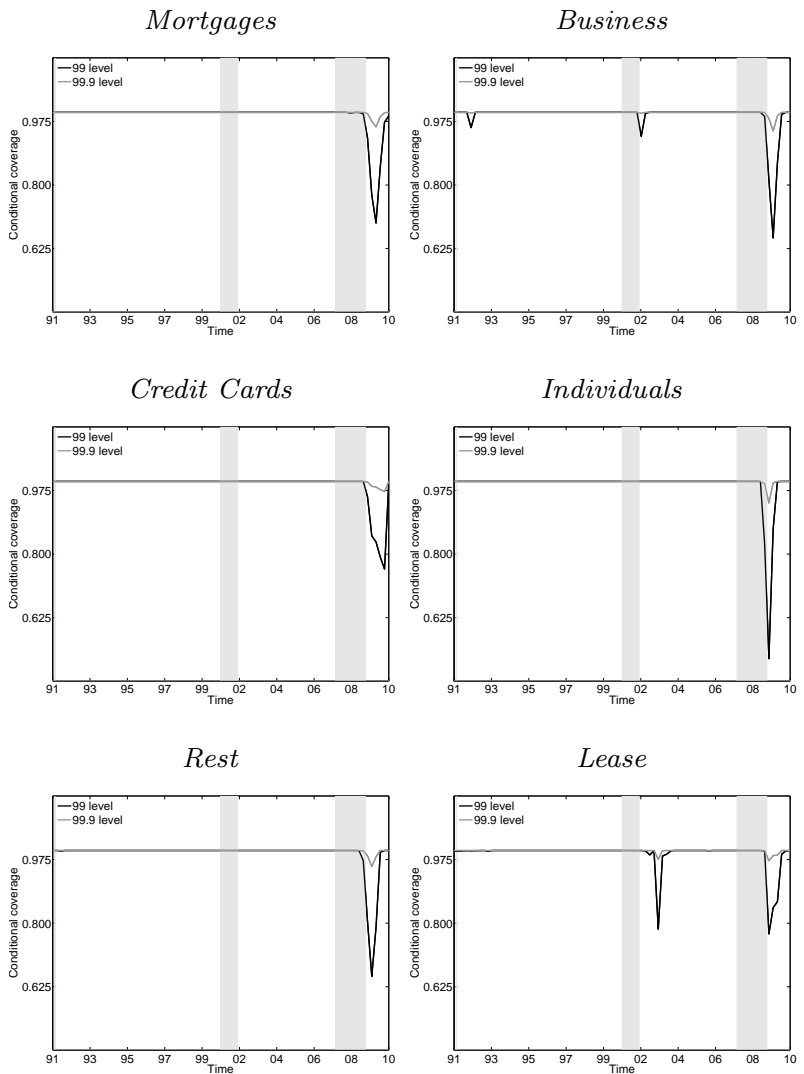

Figure 2: Conditional coverage series generated by the unconditional capital estimates for the 99 and 99.9 coverage levels. Shaded areas indicate U.S. recessions according to NBER (National Bureau of Economic Research).

identically distributed - that is, there is no cyclical behavior at all- then $L$ would be also distributed as $L_{t}$. Therefore, $u^{d t}=u$ and no increase in $\eta$ is needed regardless of the value of $u$.

Also, for example, if the time window is formed by two time periods with $L_{1}$ being uniform in $(01)$ and $L_{2}$ uniform (24), then, at $u=90, \eta=3.7$ and the corresponding minimum conditional coverage is $u^{d t}=85$. It would be necessary to consider $\eta=3.8$ to get $u^{d t}=90$; that is, an increase of $2.70 \%$ in $\eta$. However, if $L_{1}$ is uniform $\left(\begin{array}{l}0 \\ 0\end{array}\right)$ and $L_{2}$ uniform $(34)$, then, at $u=90, \eta=3.7$ too, but $u^{d t}=70$ and the required capital to get $u^{d t}=90$ would be $\eta=3.9$, which means an increase of $5.41 \%$ instead of $2.70 \%$.

Obviously, capital figures high enough so as to guarantee values of $u^{d t}$ equal to 99 or 99.9 would generate huge coverage values when interpreted in terms of the unconditional loss distribution, as can be seen in Table 6 .

To put this data into perspective, it is worth conducting the next back-of-the-envelope calculation. According to S\&P (2012), the maximum annual hazard rate observed between 1991 and 2010 in the Standard \& Poor's AA rating for corporate debtors is 0.0038 (achieved in 2008). As-
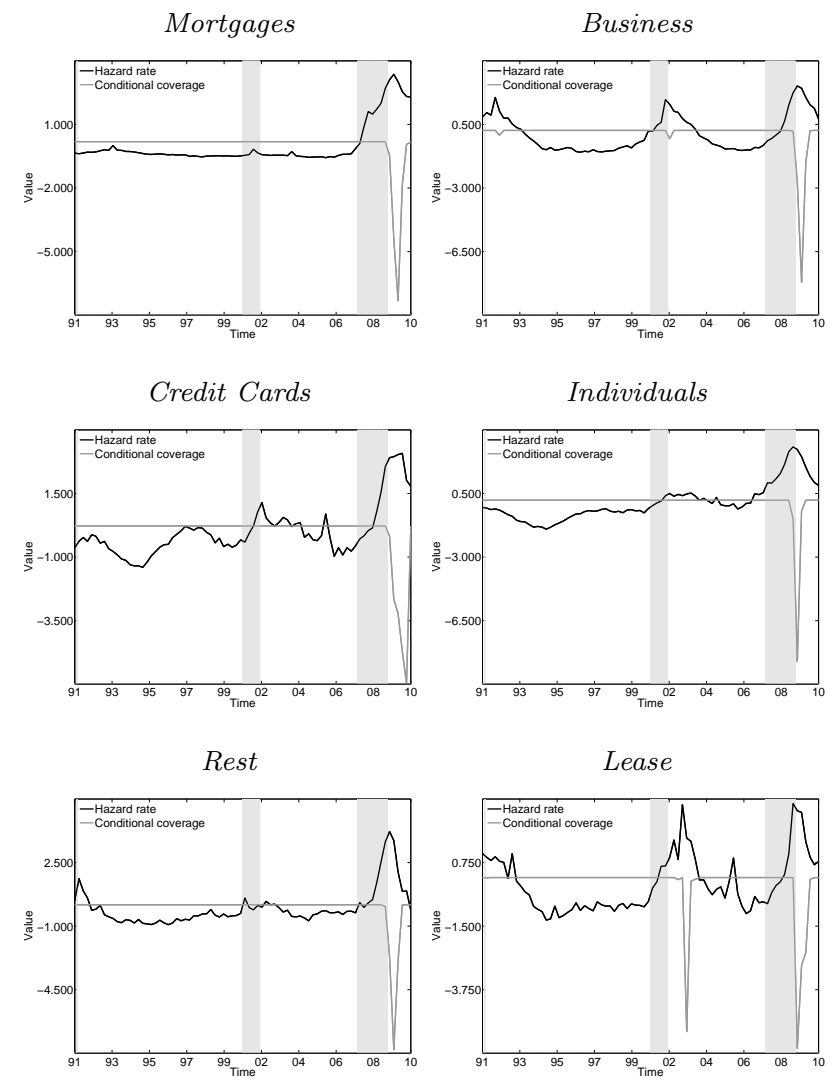

Figure 3: Hazard rate, $h_{t}$, and conditional coverage, $u_{t}$, series generated by the unconditional 99.9 capital estimate. Both series are presented standardized. Shaded areas indicate U.S. recessions according to NBER (National Bureau of Economic Research).

suming proportional hazards ${ }^{8}$, this is equivalent to 0.0011 on a quarterly basis, or approximately 0.001 . Then, following the methodology discussed in Section $3, h^{R} \approx 0.001$, which means taking $u^{d t}=99.9$. According to Table 6 , a capital estimate achieving such downturn coverage would offer an unconditional coverage ranging from $u=99.9967$ for Lease to $u=99.9985$ in the case of Individuals. On the other hand, the average of the AA corporate rating hazard rate series is 0.000275 , or approximately 0.00007 on a quarterly basis. That means an unconditional coverage $u=100-0.0070=99.9930$. Therefore, in this toy example, determining $\eta$ in terms of $u^{d t}$ through the ratingbased methodology leads to a slightly higher capital ${ }^{9}$ than that obtained in terms of $u$.

With respect to the stability of the conditional coverage values, Table 7 presents the value of $s^{\boldsymbol{u}}$ for each combination of portfolio and unconditional coverage level.

Given $u$, differences among portfolios can be observed, with Credit Cards and Lease being the most, and Individ-

\footnotetext{
${ }^{8}$ That is, $y=1-(1-x)^{0.25}$, with $x$ being the annual rate and $y$ its quarterly equivalent.

${ }^{9}$ A higher unconditional coverage requires more capital.
} 


\begin{tabular}{lcc}
\hline \multicolumn{1}{c}{ Portfolio } & $u^{d t}=99$ & $u^{d t}=99.9$ \\
\hline Mortgages & 0.3901 & 0.2780 \\
Business & 0.2476 & 0.1793 \\
Credit Cards & 0.1370 & 0.0982 \\
Individuals & 0.1328 & 0.0885 \\
Rest & 0.5572 & 0.3533 \\
Lease & 0.3773 & 0.2597 \\
\hline
\end{tabular}

Table 5: Relative increase in the capital figures of Table 3 needed to achieve a downturn coverage equal to $99, u^{d t}=$ 99, and to $99.9, u^{d t}=99.9$.

\begin{tabular}{lcc}
\hline \multicolumn{1}{c}{ Portfolio } & $u^{d t}=99$ & $u^{d t}=99.9$ \\
\hline Mortgages & 99.9752 & 99.9977 \\
Business & 99.9790 & 99.9971 \\
Credit Cards & 99.9675 & 99.9978 \\
Individuals & 99.9830 & 99.9985 \\
Rest & 99.9792 & 99.9976 \\
Lease & 99.9665 & 99.9967 \\
\hline
\end{tabular}

Table 6: Unconditional coverage achieved by the capital figures whose downturn coverage is equal to $99, u^{d t}=99$, and to $99.9, u^{d t}=99.9$.

uals the least, stable portfolios. Correspondingly, given a portfolio, the higher the $u$, the lower the $s^{u}$. In other words, increasing the unconditional coverage also guarantees increasing the conditional solvency stability, which is a result consistent with Figure 2.

Figure 4 presents, for $\eta$ obtained at $u=99.9$, the scatter plot of the pair $\left(u^{d t} s^{\boldsymbol{u}}\right)$ for each portfolio. Data are obtained from Table 4 and Table 7 . According to Section 4 and Section 5, those placed in the lower-right corner are preferable to those placed in the upper-left corner. Thus, the Credit Cards portfolio presents the best performance, with Individuals being the worst. The strong correlation between $u^{d t}$ and $s^{\boldsymbol{u}}$ observed is further evidence of the fact that the variability in $\boldsymbol{u}$ is mainly driven by the marked decrease in conditional coverage values experienced during the Great Recession.

Figure 4 also outlines the differences in resilience and stability among the seven portfolios, since both axes exhibit discrimination power. Obviously, this kind of representation cannot be obtained if the long-term assessment of a given capital figure, $\eta$, is reduced to its unconditional coverage, $u$.

\section{Concluding remarks}

The main conclusion supported by this paper is the usefulness of incorporating the conditional coverage vector into the unconditional measurement of default risk. This statement rests on the theoretical result proved and the two applications proposed.

Regarding the theoretical result, Proposition A, we have shown that, under a certain model of unconditional $\mathrm{PD}$ distribution, the average of the conditional coverage

\begin{tabular}{lcc}
\hline \multicolumn{1}{c}{ Portfolio } & $\eta_{99}$ & $\eta_{99.9}$ \\
\hline Mortgages & 0.0460 & 0.0054 \\
Business & 0.0463 & 0.0059 \\
Credit Cards & 0.0416 & 0.0045 \\
Individuals & 0.0592 & 0.0066 \\
Rest & 0.0496 & 0.0055 \\
Lease & 0.0408 & 0.0047 \\
\hline
\end{tabular}

Table 7: Standard deviation of the conditional coverage vector $\boldsymbol{u}$ generated by the capital figures of Table 3, which have been obtained at the $99, \eta_{99}$, and the $99.9, \eta_{99.9}$, unconditional coverage levels.

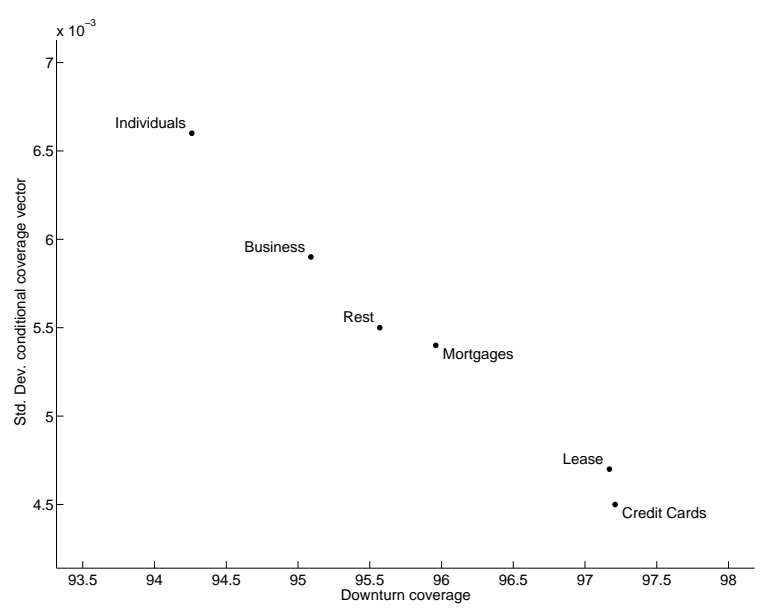

Figure 4: Downturn coverage, $u^{d t}$, displayed at the horizontal axis, and standard deviation of the conditional coverage vector, $s^{\boldsymbol{u}}$, displayed at the vertical axis. Both are derived from the conditional coverage vector generated by the 99.9 unconditional capital estimate.

values generated by a given constant capital equals the corresponding unconditional coverage. This result coherently links the concepts of conditional and unconditional solvency and allows for an alternative interpretation of the unconditional capital.

Regarding the applications, we have used the conditional coverage vector in both the long-term capital determination and assessment processes. For the former, we have proposed determining the capital in terms of the minimum of the vector instead of resorting to the unconditional coverage, which constitutes the standard approach. For the latter, we have proposed assessing the long-term solvency of a given capital, not only by means of its unconditional coverage, but also in terms of the minimum and standard deviation of the conditional coverage vector. We have illustrated both applications empirically, observing a cyclical and asymmetric pattern in the conditional coverage vector and a remarkable discriminating power in its minimum and standard deviation.

An additional remark can be drawn on the basis of these results. Since determining or assessing a long-term capital means implementing or evaluating a long-term risk 
appetite statement, our contribution indicates that the characterization of such a statement should not be left to a single variable, $u$, but it should also include $u^{d t}$ and $s^{\boldsymbol{u}}$, which complement $u$ by recognizing the cyclical nature of default risk. Therefore, they provide new possibilities for setting, communicating and monitoring risk appetite.

\section{Appendix A.}

\section{Proof of Proposition $A$.}

The proof follows directly from the elementary properties of finite mixtures, see McLachlan and Peel (2000).

Since $F^{* j}$ is the mixture of the distributions $F_{t}^{j}, t=$ $1, \ldots, T$, then $L^{j}=F^{* j} e^{j}$ is the mixture of the distributions $L_{t}^{j}=F_{t}^{j} e^{j}, t=1, \ldots, T$. This, in turn, means that $L=$ $\sum_{j=1}^{N} L^{j}$ is the mixture of the distributions $L_{t}=\sum_{j=1}^{N} L_{t}^{j}$, $t=1, \ldots, T$.

On the other hand, the cumulative distribution function of an equally weighted mixture is the average of the cumulative distribution function of its components, so that

$$
P(L \leq x)=\frac{1}{T} \sum_{t=1}^{T} P\left(L_{t} \leq x\right)
$$

for any $0<x<\sum_{j=1}^{N} e^{j}$, and therefore

$$
u=P(L \leq \eta)=\frac{1}{T} \sum_{t=1}^{T} P\left(L_{t} \leq \eta\right)=\frac{1}{T} \sum_{t=1}^{T} u_{t}
$$

\section{References}

Ayuso, J., Pérez, D., Saurina, J., 2004. Are capital buffers procyclical? Evidence from spanish panel data. Journal of Financial Intermediation 13 (2), 249-264.

BCBS, 2006. Basel II: International convergence of capital measurement and capital standards - revised version. Bank for International Settlements.

BCBS, 2011. Basel III: A global regulatory framework for more resilient banks and banking systems - revised version. Bank for International Settlements.

BCBS, 2013. Basel III: International framework for liquidity risk measurement, standards and monitoring - revised version. Bank for International Settlements.

Carey, M., 2002. A guide to choosing absolute bank capital requirements. Journal of Banking \& Finance 26 (5), 929-951.

Drumond, I., 2009. Bank capital requirements, business cycle fluctuations and the Basel accords: A synthesis. Journal of Economic Surveys 23 (5), 798-830.

Duffie, D., Saita, L., Wang, K., 2007. Multi-period corporate default prediction with stochastic covariates. Journal of Financial Economics 83 (3), 635-665.

Elizalde, A., Repullo, R., 2007. Economic and regulatory capital in banking: What is the difference? International Journal of Central Banking 3 (3), 87-117.

Estrella, A., 2004. The cyclical behavior of optimal bank capital. Journal of Banking \& Finance 28 (6), 1469-1498.

Ferrer, A., Casals, J., Sotoca, S., 2014. A new approach to the unconditional measurement of default risk. ICAE Working Papers.
Festic, M., Kavkler, A., Repina, S., 2011. The macroeconomic sources of systemic risk in the banking sectors of five new E.U. member states. Journal of Banking \& Finance 35 (2), 310-322.

Foglia, A., 2009. Stress testing credit risk: A survey of authorities' approaches. International Journal of Central Banking 5 (3), 9-46.

Frey, R., McNeil, A., 2003. Dependent defaults in models of portfolio credit risk. The Journal of Risk 6, 59-92.

Glasserman, P., 2004. Tail approximations for portfolio credit risk. The Journal of Derivatives 12 (2), 24-42.

Glasserman, P., Kang, W., Shahabuddin, P., 2008. Fast simulation of multifactor portfolio credit risk. Operations Research 56 (5), 1200-1217.

Gordy, M., 2000. A comparative anatomy of credit risk models. Journal of Banking \& Finance 24 (1-2), 119-149.

Gordy, M., 2003. A risk-factor model foundation for ratings-based bank capital rules. Journal of Financial Intermediation 12 (3), 199-232.

Gordy, M., Howells, B., 2006. Procyclicality in Basel II: Can we treat the disease without killing the patient? Journal of Financial Intermediation 15 (3), 395-417.

Heid, F., 2007. The cyclical effects of the Basel II capital requirements. Journal of Banking \& Finance 31 (12), 3885-3900.

Jiménez, G., Mencía, J., 2009. Modelling the distribution of credit losses with observable and latent factors. Journal of Empirical Finance 16 (2), 235-253.

Jokipii, T., Milne, A., 2008. The cyclical behaviour of european bank capital buffers. Journal of Banking \& Finance 32 (8), 1440-1451.

Kashyap, A. K., Stein, J. C., 2004. Cyclical implications of the Basel II capital standards. Economic Perspectives-Federal Reserve Bank Of Chicago 28 (1), 18-33.

Koopman, S., Lucas, A., Klaassen, P., 2005. Empirical credit cycles and capital buffer formation. Journal of Banking \& Finance 29 (12), 3159-3179.

McLachlan, G., Peel, D., 2000. Finite mixture models. WileyInterscience.

Pesaran, M., Schuermann, T., Treutler, B., Weiner, S., 2006. Macroeconomic dynamics and credit risk: a global perspective. Journal of Money, Credit, and Banking 38 (5), 1211-1261.

Peura, S., Jokivuolle, E., 2004. Simulation based stress tests of banks' regulatory capital adequacy. Journal of Banking \& Finance 28 (8), 1801-1824.

Repullo, R., 2013. Cyclical adjustment of capital requirements: A simple framework. Journal of Financial Intermediation 22 (4), $608-626$.

Repullo, R., Saurina, J., Trucharte, C., 2010. Mitigating the procyclicality of Basel II. Economic Policy 25 (64), 659-702.

Rösch, D., Scheule, H., 2010. Downturn credit portfolio risk, regulatory capital and prudential incentives. International Review of Finance 10 (2), 185-207.

Sorge, M., Virolainen, K., 2006. A comparative analysis of macro stress-testing methodologies with application to Finland. Journal of Financial Stability 2 (2), 113-151.

S\&P, March 2012. 2011 annual global corporate default study and rating transitions. Standard \& Poor's Global Fixed Income Research.

Truck, S., Rachev, S. T., 2005. Credit portfolio risk and probability of default confidence sets through the business cycle. The Journal of Credit Risk 1 (4), 61-88.

Varotto, S., 2012. Stress testing credit risk: The great depression scenario. Journal of Banking \& Finance 36 (12), 3133-3149.

Vasicek, O., 2002. The distribution of loan portfolio value. Risk 15 (12), 160-162.

Zhu, H., 2008. Capital regulation and banks' financial decisions. International Journal of Central Banking 4 (1), 165-212. 